\title{
Detrimental complications due to improper antithrombotic management in the preoperative period: report of two cases
}

\author{
Sung-Hye Byun, Byungdoo Son, and Jong Chan Kim \\ Department of Anesthesiology and Pain Medicine, Kyungpook National University Hospital, Daegu, Korea
}

Antiplatelet agents, including aspirin and clopidogrel, and anticoagulants are widely used for the primary or secondary prevention of cardiovascular events, as well as for other therapeutic purposes. However, they tend to be interrupted before surgery to reduce the risk of bleeding in many patients. We encountered two cases of patients who experienced myocardial infarct (MI) or cerebrovascular events in the postoperative period due to improper antithrombotic management before surgery.

The first patient was a 75-year-old man scheduled to undergo open partial nephrectomy for renal cell carcinoma. He took an angiotensin receptor blocker for hypertension and had voluntarily stopped taking aspirin several months earlier. Preoperative electrocardiography (ECG) revealed T-wave inversion in leads I, aVL, V5-6, consistent with an interval change compared with previous normal ECG. Transthoracic echocardiography (TTE) and myocardial perfusion scan showed left ventricular ejection fraction (LVEF) of 53\% and regional wall motion abnormalities (RWMAs) in left anterior descending (LAD) and left circumflex (LCX) territories; these RWMAs had not been observed on TTE taken 3 years earlier. Cardiac intervention was recommended before surgery, but the patient strongly refused this recommendation from private reason. Because he did not present with symptoms of chest pain or dyspnea, surgery first planned, followed by timely postoperative administration of aspirin. After surgery was achieved successfully without any abnormal events, spontaneous respiration was recovered and the endotracheal tube was extubated. However, the patient complained of dyspnea on post-anesthetic care unit. After the patient was moved to intensive care unit receiving oxygen $4 \mathrm{~L} / \mathrm{min}$ through a mask, arterial blood gas analysis values were within normal range. However, postoperative cardiac enzymes showed increased levels of cardiac troponin I (cTnI, $3.51 \mathrm{ng} / \mathrm{ml}$ ) and creatine kinase MB $(0.5 \mathrm{ng} / \mathrm{ml})$. TTE showed a reduced LVEF (30\%) and new RWMAs. Therefore, postoperative acute MI and dyspnea due to left ventricular dysfunction were suspected and intravenous nitrate was infused. On postoperative day (POD) 3, nitrate infusion was stopped and intravenous heparinization was initiated. After confirming a decrease in the cTnI level to 0.789 $\mathrm{ng} / \mathrm{ml}$, heparin administration was stopped on POD 8. Follow-up TTE revealed LVEF 33\% and RWMAs in LAD/LCX territories without interval change. Anti-anginal drugs, including aspirin, were prescribed, and the patient was discharged without further problems on POD 13.

The second patient was a 44-year-old woman presented with right lower abdominal pain to gynecologist. Abdominal computed tomography (CT) showed bilateral enlargement of the ovaries with peritoneal carcinomatosis. She had no previous underlying disease or history of surgery. However, the patient had signs of ascites, bilateral pleural effusion, and dyspnea. Preoperative ECG and TTE showed sinus tachycardia (120 beats/min) and moderate hypokinesia of enlarged right ventricle with normal LVEF, respectively. Because CT showed deep vein thrombosis (DVT) in the anterior and posterior tibial veins in both legs, pulmonary thromboembolism (PTE) in the right lower lobar artery, she received subcutaneous injection of low-molecular weight heparin (LMWH). Diagnostic exploration surgery was planned urgently because abdominal pain aggravated suddenly. However, at that time, anticoagulation had been discontinued

Corresponding author: Jong Chan Kim, M.D., Department of Anesthesiology and Pain Medicine, Kyungpook National University Hospital, 130, Dongdeok-ro, Jung-gu, Daegu 700-721, Korea. Tel: 82-53-200-3494, Fax: 82-53-426-2760, E-mail: aescula72@hanmail.net

(c) This is an open-access article distributed under the terms of the Creative Commons Attribution Non-Commercial License (http:// creativecommons.org/licenses/by-nc/3.0/), which permits unrestricted non-commercial use, distribution, and reproduction in any medium, provided the original work is properly cited. 
for 3 days because of anemia by hematuria. After surgery was achieved uneventfully, antithrombotics were restarted on POD 3. However, warfarin was unintelligibly stopped again for chemoport insertion on POD 10. On POD 14, the patient experienced neurologic symptoms including headache, temporary loss of consciousness and developed left limb weakness. Brain magnetic resonance imaging showed multiple bihemispheric cerebral infarctions and occlusion of both right and left middle cerebral arteries and posterior cerebral arteries. Although intravenous thrombolytic therapy was started after neurologic consultation, follow-up brain CT still showed multifocal cerebral infarction. She was transferred to other hospital with stuprous mentality on POD 16.

Perioperative antiplatelet or anticoagulant therapy, the timing of discontinuation of these drugs, and bridging these therapies in surgical patients warrants attention because of the cardiovascular complications resulting from the preoperative discontinuation of these drugs. Although many guidelines address this concern, the attending physicians should decide whether these therapies should be discontinued preoperatively to reduce the risk of hemorrhage or continued to prevent the risk of MI or cerebrovascular events in the perioperative period. However, this decision may be difficult because perioperative management of anticoagulant and antiplatelet therapies is complicated, and should be individualized. Several recent guidelines recommend that these therapies should be maintained perioperatively, especially in high-risk patients $[1,2]$.

The first patient had a history of hypertension and cerebrovascular event, but had voluntarily stopped taking aspirin several months earlier. Although preoperative cardiac evaluation revealed new RWMAs that had developed within 3 years, antiplatelet drug was not administrated preoperatively, which might have worsened heart function. Low-dose aspirin has been associated with a 1.5-fold intraoperative blood loss compared with controls who were not administered aspirin, with no increase in hemorrhage-associated mortality or morbidity [3]. In addition, rupture of an unstable plaque followed by thrombosis is the main cause of postoperative MI, and this type of infarction occurs early within $36 \mathrm{~h}$ in the postoperative period, and is prevented by antiplatelet drugs [4].

The second patient had PTE due to DVT and secondary right heart failure due to PTE. Nevertheless, anticoagulation was discontinued twice for various reasons. According to the American College of Chest Physicians guidelines [2], she was a high-risk patient and should have been managed with bridging therapy with anticoagulants to prevent catastrophic cerebrovascular events. In an observational study, the relative risk of reduction for thromboembolism with bridging therapy was estimated to be $66-80 \%$. Therefore, if a patient's risk of thromboembolism is $1.5 \%$, bridging therapy attenuates the risk to $\leq 0.5 \%$ [5].

The concept that the practice of withdrawing antiplatelet drugs 5-10 days prior to surgical procedures should be changed, and that bridging anticoagulation therapy is needed to prevent cardiovascular events perioperatively, especially in high-risk patients, has gained widespread acceptance. As mentioned previously, appropriate perioperative management with antithrombotic therapy is very complicated, and should be adequately discussed by all attending physicians, including anesthesiologists, cardiologists and surgeons, considering patients' characteristics, surgical risks, and other factors. Adequate consideration and application of these therapies are mandatory to prevent deleterious perioperative cardiovascular complications and improve patients' quality of life.

\section{References}

1. Fleisher LA, Beckman JA, Brown KA, Calkins H, Chaikof EL, Fleischmann KE, et al. ACC/AHA 2007 Guidelines on Perioperative Cardiovascular Evaluation and Care for Noncardiac Surgery: Executive Summary: A Report of the American College of Cardiology/ American Heart Association Task Force on Practice Guidelines (Writing Committee to Revise the 2002 Guidelines on Perioperative Cardiovascular Evaluation for Noncardiac Surgery) Developed in Collaboration With the American Society of Echocardiography, American Society of Nuclear Cardiology, Heart Rhythm Society, Society of Cardiovascular Anesthesiologists, Society for Cardiovascular Angiography and Interventions, Society for Vascular Medicine and Biology, and Society for Vascular Surgery. J Am Coll Cardiol 2007; 50: 1707-32.

2. Douketis JD, Berger PB, Dunn AS, Jaffer AK, Spyropoulos AC, Becker RC, et al. The perioperative management of antithrombotic therapy: American College of Chest Physicians Evidence-Based Clinical Practice Guidelines (8th Edition). Chest 2008; 133: S299-339.

3. Burger W, Chemnitius JM, Kneissl GD, Rücker G. Low-dose aspirin for secondary cardiovascular prevention - cardiovascular risks after its perioperative withdrawal versus bleeding risks with its continuation - review and meta-analysis. J Intern Med 2005; 257: 399-414.

4. Chassot PG, Delabays A, Spahn DR. Perioperative use of anti-platelet drugs. Best Pract Res Clin Anaesthesiol 2007; 21: 241-56.

5. Kearon C, Hirsh J. Management of anticoagulation before and after elective surgery. N Engl J Med 1997; 336: 1506-11. 